\title{
Alter
}

Revue de phénoménologie

$28 \mid 2020$

La religion

\section{Paul Ricœur, ou la religion sous epokhê}

\section{Daniel Frey}

\section{(2) OpenEdition}

\section{Journals}

Édition électronique

URL : https://journals.openedition.org/alter/2026

DOI : $10.4000 /$ alter.2026

ISSN : 2558-7927

\section{Éditeur :}

Association ALTER, Archives Husserl (CNRS-UMR 8547)

\section{Édition imprimée}

Date de publication : 1 novembre 2020

Pagination : 133-148

ISBN : 978-2-9550449-6-4

ISSN : 1249-8947

\section{Référence électronique}

Daniel Frey, «Paul Ricœur, ou la religion sous epokhê », Alter [En ligne], 28 | 2020, mis en ligne le 22 décembre 2020, consulté le 15 octobre 2021. URL : http://journals.openedition.org/alter/2026 ; DOI : https://doi.org/10.4000/alter.2026

Ce document a été généré automatiquement le 15 octobre 2021

Revue Alter 


\title{
Paul Ricœur, ou la religion sous epokhê
}

\author{
Daniel Frey
}

Dans Du texte à l'action, Paul Ricœur avait caractérisé de façon précise son ancrage dans la tradition philosophique en le situant « dans la ligne d'une philosophie réflexive [...] », et plus particulièrement dans la mouvance de la phénoménologie husserlienne » dont sa propre philosophie constituait à ses yeux une "variante herméneutique $»^{1}$. Le rapport à la phénoménologie de Husserl remonte aux origines même de son œuvre, puisque Le Volontaire et l'Involontaire - sa thèse de doctorat soutenue en 1948 et parue en 1950 cherchait déjà à honorer une triple fidélité à Edmund Husserl, à Gabriel Marcel et à Karl Jaspers. Ricœur se réclamait du premier pour son exigence de rigueur méthodologique, et devait aux deux autres de situer toute pensée de l'existence en face de la Transcendance. Désireux de faire converger ces trois influences disparates, le jeune philosophe s'efforçait d'élaborer une philosophie existentielle aimantée par une Transcendance qui d'emblée nécessitait d'être mise entre parenthèses pour des questions de méthode. Or les Ideen de Husserl (que Ricœur a traduites au titre de thèse secondaire) lui fournissaient le modèle d'une telle epokhê, désignée précisément par Husserl comme une "mise "entre parenthèses" ou hors circuit» [Einklammerung, Ausschaltung] :

Par rapport à chaque thèse nous pouvons, avec une entière liberté, opérer cette غ̇ंoxń originale, c'est-à-dire une certaine suspension du jugement qui se compose avec une persuasion de la vérité qui demeure inébranlée [...] La «thèse » est « mise hors de jeu », entre parenthèses: elle se convertit dans la forme modifiée : «thèse entre parenthèses $»^{2}$.

2 On sait que cette réduction phénoménologique devait permettre de suspendre l'attitude naturelle caractérisée par la croyance (au sens général d'un tenir-pour-vrai) en l'extériorité des choses, ce qui est le seul moyen pour la conscience de découvrir son pouvoir constituant à l'égard des phénomènes; l'étude phénoménologique ayant ensuite pour tâche de faire le tour des possibilités de donations du phénomène, selon la méthode de la variation éidétique ${ }^{3}$. Husserl lui-même avait étendu l'epokhê à Dieu : dans le paragraphe des Ideen intitulé «La Transcendance de Dieu mise hors circuit», il 
rappelle que l'attitude naturelle à l'égard du monde peut conduire à admettre une Transcendance vis-à-vis du monde et de la conscience: "Cet "absolu", ce "transcendant" rentre naturellement dans la réduction phénoménologique. Il doit être exclu du nouveau champ d'étude qu'il nous faut instituer dans la mesure où ce doit être le champ de la conscience pure $»^{4}$.

En suivant de la Philosophie de la volonté à l'Essai sur Freud les quelques références où Paul Ricœur renvoie à l'epokhê husserlienne, il doit être possible de se faire une idée assez juste de son approche philosophique de la religion. C'est du moins le pari du présent essai, prélude d'une étude en cours de parution portant sur l'ensemble des écrits que Ricœur a consacrés à la religion, qu'il tenait pour le premier autre de la philosophie.

\section{Une singulière abstraction : la mise entre parenthèses de la Transcendance}

Premier tome d'une Philosophie de la volonté qui aurait dû en compter trois, Le Volontaire et l'Involontaire est une description eidétique de la volonté directement inspirée de la méthode phénoménologique de Husserl. Dès les premières lignes, Ricœur signale que sa description pure des structures de la volonté humaine - autrement dit des « possibilités fondamentales de l'homme »- doit être entreprise sans aucun égard pour la notion de "Transcendance ", dont il rappellera pourtant à plusieurs reprises dans l'introduction qu'elle est constitutive de toute pensée sur la subjectivité ${ }^{5}$. Le Volontaire et l'Involontaire s'ouvre plus précisément sur une double abstraction, qui a tout d'une soustraction: la Transcendance et la faute - expression laïcisée de Dieu et du péché - sont soustraites à l'examen comme il se doit dans une étude phénoménologique :

L'étude des rapports entre Le Volontaire et l'Involontaire forme la première partie d'un ensemble plus vaste qui porte le titre général de "Philosophie de la volonté ». Les problèmes qui sont abordés dans cet ouvrage et la méthode qui y est mise en œuvre sont donc délimités par un acte d'abstraction qu'il est nécessaire de justifier dans cette introduction; les structures fondamentales $\mathrm{du}$ volontaire et de l'involontaire que l'on cherche ici à décrire et à comprendre ne recevront leur signification définitive que lorsque l'abstraction qui en a permis l'élaboration aura été levée. C'est en effet en mettant entre parenthèses la faute, qui altère profondément l'intelligibilité de l'homme, et la Transcendance qui recèle l'origine radicale de la subjectivité, que se constitue une description pure et une compréhension du Volontaire et de l'Involontaire 6 .

Dans cette entrée en matière, Ricœur court le risque de désorienter d'emblée son lecteur en anticipant un acte d'abstraction qui n'est compréhensible qu'au terme de l'introduction, voire au terme de la Philosophie de la volonté tout entière. Le philosophe deviendra coutumier de ce genre d'annonce anticipatrice qui ne prend réellement sens qu'à la relecture. Le risque pris dit bien l'importance de l'enjeu : le philosophe se doit de justifier d'emblée cette double abstraction qui est étrange à ses propres yeux puisqu'elle contrevient à l'héritage revendiqué de Gabriel Marcel et de Karl Jaspers. Ricœur a en effet hérité d'eux une conception spécifique de la réflexion philosophique, répartie autour de deux pôles tout à la fois distincts et reliés, l'existence et la Transcendance. Bien qu'il ne soit pas autrement défini, le concept de Transcendance lui permet de désigner l'Être transcendant auquel le sujet humain doit son fondement et son être, tout en évitant délibérément toute nomination particulière du divin. De fait, Ricœur propose une généralisation du vécu religieux marquant une distance certaine 
vis-à-vis de la tradition chrétienne. Le terme de «faute », qui fait sans doute écho à la "Schuld" thématisée par Jaspers, manifeste le même souci de généraliser la notion chrétienne de péché. À la même époque, une réflexion de type existentialiste pouvait bien évidemment avoir comme seul pôle l'existence, comme dans l'existentialisme athée de Sartre. C'est donc pour prévenir son lecteur de la spécificité de sa propre approche que Ricœur prend soin de préciser d'emblée que sa description pure du volontaire et de l'involontaire est incomplète tant qu'elle n'a pas été référée à la Transcendance. De son tout premier ouvrage intitulé Gabriel Marcel et Karl Jaspers Philosophie du mystère et philosophie du paradoxe ${ }^{7}$, Ricœur a retenu que l'existence doit être transcendée : le sujet est transcendé par tout ce qui le fonde et qu'il ne crée pas. Sans le transcendant visant tout ce qu'il n'est pas, le sujet n'est pas totalement lui-même: transcender est ce qui ne peut manquer d'arriver à un sujet qui est situé dans l'être ; autrement dit qui ne s'est pas donné l'existence mais l'a reçue comme un don. La polarité Existence/Transcendance sous-tend ainsi l'ensemble du projet de la Philosophie de la volonté, que le philosophe se représentait alors comme un ternaire commençant par la description pure dans Le Volontaire et l'Involontaire, poursuivi par une " description empirique de la faute $»^{8}$ et couronné enfin par une "Poétique de la liberté $»^{9}$ qui ne sera jamais rédigée.

Mais pour l'heure, une fois passé l'incipit du Volontaire et l'involontaire, le lecteur ne trouvera aucune justification du concept de Transcendance : il retrouvera seulement dans l'introduction une nouvelle anticipation des développements futurs, qui d'ailleurs resteront à jamais à venir puisqu'ils ne seront jamais rédigés : « La Transcendance nous apparaîtra plus tard comme une position absolue de présence qui sans cesse précède mon pouvoir propre d'affirmation, bien que celui-ci semble toujours sur le point de l'englober $\aleph^{10}$. La référence à la polarité Existence/Transcendance, bien qu'aussi laïque que possible dans le contexte des années 50 marquées par l'opposition des existentialismes athées et chrétiens, est en fait sous-tendue chez Ricœur par une perspective religieuse, chrétienne en l'occurrence : les écrits chrétiens militants ${ }^{11}$ que le philosophe publie en marge de son activité professorale laïque ${ }^{12}$ souligneront ainsi la spécificité de la dépendance du sujet à l'égard d'un Être divin transcendant.

Une lecture attentive de la thèse de Ricœur (impossible à produire ici) permettrait de montrer que la problématique de l'involontaire et du volontaire culmine dans le thème d'une vie reçue comme un don et vécue comme une liberté, en affinité avec une conception luthérienne de l'existence devant Dieu. La suite de la Philosophie de la volonté permettra à Ricœur de livrer une lecture plus précise du phénomène religieux, soumis là encore à une epokhê particulière.

\section{Neutralisation de l'acte de foi et prééminence des symboles bibliques}

8 Intitulée Finitude et culpabilité, cette suite est elle-même divisée en deux volumes: L'Homme faillible et La Symbolique du mal. De la même façon que Le Volontaire et l'Involontaire corrélait d'emblée l'éclairement de l'existence à l'abstraction de la faute et de la Transcendance, l'approche philosophique du religieux se rend solidaire de la question des symboles du sacré et des mythes. Ce questionnement est mis en œuvre tant dans l'introduction de l'ouvrage ("Phénoménologie de l'“aveu”») que sa conclusion ("Le symbole donne à penser») qui constituent deux textes en miroir. L'introduction 
affirme d'entrée de jeu la capacité du discours philosophique à la «reprise » ou la " répétition" de l'aveu que "la conscience religieuse $»^{13}$ fait de son passage de la faillibilité à la faute, reprise/répétition que Ricœur rattache dans la conclusion au vœu philosophique (hérité de Lachelier) de « tout comprendre, même la religion $»^{14}$.

Ce qui est essentiel ici, c'est que Ricœur prenne soin de préciser que la reprise visée est une "répétition en imagination et en sympathie", donc sur un mode neutralisé: comprenez qu'elle «n'est déjà plus religion vécue et [...] pas encore philosophie »" Cette insistance sur la neutralisation de l'expérience religieuse, Ricœur l'avait déjà élevée au rang de principe méthodologique énoncé dans une terminologie phénoménologique, dans l'essai synthétique de 1953 intitulé «Culpabilité tragique et culpabilité biblique » :

[...] l'application de la méthode comparative aux mythes grecs et aux mythes hébraïques a pour condition la neutralisation de l'acte de foi qui, éventuellement, pourrait rattacher ces mythes à un acte rédempteur qui me concerne, qui nous concerne; l'élection d'Israël, le Credo de l'Église chrétienne concernant le second Adam subissent une sorte de réduction phénoménologique par laquelle l'intention transformante qui va du mythe païen au mythe hébraïque est retenue, mais sans que l'historien y prenne part; le mythe retenu dans sa structure et dans son intention devient ainsi un phénomène culturel signifiant. Cet artifice méthodologique - car c'est un artifice, comme toutes les sciences en pratiquent lorsqu'elles élaborent leur «objet» - permet désormais une comparaison homogène des mythes sur le plan d'une unique phénoménologie. C'est la même réduction phénoménologique que nous opérons sur la religion dionysiaque qui anima de son «enthousiasme» le spectacle tragique : nous revivons, sur un mode neutralisé, la croyance dionysiaque, lorsque la grâce tragique nous touche, par la terreur et la pitié, et que nous nous livrons à ce savoir douloureux, mais pacifié par la poésie, que la tragédie communique ${ }^{16}$.

\section{La réduction phénoménologique est ici la suspension de l'assentiment, l'epokhê}

husserlienne déjà évoquée dans l'introduction : en affirmant que le préalable à l'étude comparée des mythes est la mise en suspens de l'acte de foi par lequel le sujet pourrait relier le mythe à un acte de salut, Ricœur formalise une règle méthodologique essentielle à l'herméneutique du langage symbolique qu'il entreprend. On notera en

l'acte de foi

outre que si

est suspendu, la reconnaissance de « l'intention transformante » des mythes et discours religieux à l'égard des auditeurs doit être reconnue et vécue, mais « sur un mode neutralisé »

17

. Loin d'être une inconséquence, cette distinction exprime toute la différence entre

l'attitude scientifique, dont Ricœur a dit ailleurs qu'elle aussi« procède d'une certaine "réduction" de notre rapport total au monde total; un seul aspect du monde est retenu: son aspect quantitatif et mesurable $»^{18}$, et l'attitude phénoménologique. En effet, s'il suffit de suspendre toute foi dans la réalité qu'évoque le mythe religieux pour en faire une étude scientifique, il faut, pour en proposer une analyse phénoménologique ${ }^{19}$, s'ouvrir à l'intention religieuse traduite par les symboles et les récits qui les emploient. La réduction phénoménologique qu'opère le philosophe sur l'acte de foi est donc la condition d'une approche distanciée des mythes religieux, étendue bien au-delà de la sphère judéo-chrétienne jusqu'à inclure la religion babylonienne et la tragédie religieuse grecque. 
11 Malgré cette extension, Ricœur n'en reconnaît pas moins la relation privilégiée que sa pensée entretient avec les symboles bibliques du mal, auxquels il accorde une prééminence parmi les autres symboles. Il entend bien la justifier dès l'introduction, en notant d'une part que «la source juive est le premier "autre" de la philosophie, son autre le plus "proche"; le fait abstraitement contingent de cette rencontre est le destin même de notre existence occidentale $»^{20}$ - et en rappelant d'autre part, contre toute prétention à " échapper à cette contingence des rencontres historiques et se tenir hors du jeu au nom d'une "objectivité" non située $»^{21}$, qu'il n'y a de recherche qu'ancrée dans une situation historique donnée. Il n'y a pas d'écoute possible de tous les mythes sans qu'une première écoute (de la Bible en l'occurrence) ait été opérée, qui aura toujours la préférence du philosophe, même si elle reste toujours marquée du sceau de la contingence. Ricœur a constamment affirmé qu'une écoute située est la condition de possibilité d'une pensée du religieux. En ce sens, il s'agit moins d'une limite de la philosophie de Ricœur face à la religion qu'une limite (si l'on veut parler négativement) de la philosophie de la religion elle-même.

Plus généralement et au-delà même de la question de la religion, il n'y a pas de " philosophie sans présuppositions $»^{22}$, car il n'y a pas de recherche qui ne soit mue par une forme de pré-compréhension de ce qu'elle cherche : le fameux cercle donnant qu'il "faut comprendre pour croire, mais il faut croire pour comprendre» que Ricœur thématisera dans la conclusion ${ }^{23}$, s'inscrit lui-même dans le cercle plus vaste de la relation que tout chercheur entretient avec l'objet recherché. Faut-il faire remonter ce thème du chercheur impliqué dans sa recherche jusqu'à Être et temps de Heidegger ? On sait en effet que ce dernier relevait que l'enquête ontologique est rendue possible par le fait que le Dasein est l'étant s'interrogeant sur l'être et se mouvant dans une certaine entente préalable de l'être. Plaide en faveur de cette hypothèse le fait que Ricœur repousse l'accusation de «cercle vicieux, encore moins mortel $»^{24}$ tout comme Heidegger l'avait fait en $1927^{25}$.

13 Être situé pour comprendre et ne pouvoir interroger l'être qu'à partir d'une compréhension préalable n'est que la première partie de la justification de la préférence accordée aux symboles bibliques. La seconde consiste à vérifier dans la confrontation même des symboles religieux le rang éminent des symboles issus de la Bible. Assez logiquement, ce n'est donc qu'après avoir présenté les symboles primaires (souillure, péché, culpabilité) dans la première partie de l'ouvrage, puis les mythes de commencement et de fin dans la seconde, que Ricœur peut assumer dans le dernier chapitre l'ensemble de sa présupposition méthodologique :

La présupposition de mon entreprise, c'est que le lieu où l'on peut le mieux écouter, entendre et comprendre l'instruction des mythes dans leur ensemble, c'est le lieu où est proclamé, encore aujourd'hui, la prééminence d'un de ces mythes, le mythe adamique $^{26}$.

L'interprétation ricœurienne se sent redevable à l'égard de tous les mythes du mal (« tous nous parlent en quelque façon $»^{27}$ ), mais c'est bien le mythe biblique de la chute qui a suscité le vœu d'une " "répétition" de l'expérience que le mythe explicite »"${ }^{28}$. L'expérience en question est celle d'une liberté fondamentale mais radicalement aliénée, "énigme de la liberté serve ${ }^{29}$ tenant tout entière dans l'oxymore du serf arbitre. L'ouvrage tente évidemment de relier entre eux les différents symboles du mal, en montrant comment le mythe adamique, opposé à tous les autres, "réaffirme à des degrés variables l'essentiel des autres mythes ${ }^{30}$. Mais c'est de toute évidence à partir du mythe biblique que Ricœur a pu caractériser la conscience religieuse comme avouant 
simultanément la « liberté » et la «servitude » de la condition humaine. Encore faut-il, pour être enseigné par cette logique symbolique totalement étrangère à la logique de non-contradiction aristotélicienne, se détourner de la forme rationalisée de l'expérience religieuse de la faute, en l'occurrence de ce "péché originel » dénoncé dès la deuxième page de l'ouvrage ${ }^{31}$. Ricœur amplifiera considérablement cette critique initiale l'année même de parution de l'ouvrage, dans une leçon d'ouverture à la Faculté Libre de théologie protestante du boulevard Arago intitulée «Le péché originel: étude de signification $»^{32}$. Dans cette lecture magistrale, qui d'ailleurs a durablement marqué le champ théologique chrétien, le philosophe se propose de "défaire ${ }^{33}$ le péché originel, tâche qui présente deux dimensions: la première relève d'une approche historique scrupuleuse rappelant la déconstruction, conçue comme une méthode d'appropriation de l'histoire de la philosophie; la seconde relevant de façon plus engagée encore d'une destruction:

Je pense qu'il faut détruire le concept comme concept pour comprendre l'intention du sens : le concept de péché originel est un faux savoir et il doit être brisé comme savoir; savoir quasi-juridique de la culpabilité des nouveau-nés, savoir quasibiologique de la transmission d'une tare héréditaire, faux savoir qui bloque dans une notion inconsistante une catégorie juridique de dette et une catégorie biologique d'héritage ${ }^{34}$.

La leçon reconstitue ainsi la généalogie de ce pseudo-concept chez un Augustin tout d'abord fidèle à la tradition pénitentielle biblique venue de son maitre Ambroise, et refusant en conséquence le mal positif des Gnostiques, puis à son tour auteur d'une dogmatisation quasi-gnostique dans son opposition à Pélage et au pélagianisme. Ce travail de déconstruction est l'étape indispensable permettant de bien comprendre le symbole que le péché originel - malgré le raidissement théologique qu'il constitue continue de viser ; Ricœur allant d'ailleurs jusqu'à écrire qu'« il faut passer par l'échec $\mathrm{du}$ savoir pour retrouver l'intention orthodoxe, le sens droit, le sens ecclésial $»^{35}$. Restituer au serf arbitre son caractère symbolique permettra d'ailleurs de bien faire comprendre en quoi la régénération est elle aussi symbolique, c'est-à-dire fondée sur la vertu transformante des symboles bibliques: "si le mal est au niveau radical de la "génération", en un sens symbolique et non factuel, la conversion elle-même est "régénération" $»^{36}$. Ajoutons enfin que le principe méthodologique qui l'incite à repousser la fausse rationalisation du péché originel interdit également au philosophe de se livrer à une interprétation "allégorisante ${ }^{37} \mathrm{du}$ mythe biblique, puisqu'elle rendrait caduc le symbole de la captivité une fois son interprétation opérée.

\section{Les symboles et le sacré}

Les symboles du mal sont pour Ricœur des symboles du sacré. Il importe précisément de comprendre que ce que le symbole donne à penser n'est pas du tout une pensée, mais plutôt ce que le philosophe appelle " en des termes intentionnellement vagues ${ }^{38}$ le « lien de l'homme à son sacré $~_{39}$. Le concept de sacré, déjà employé de longue date en histoire des religions, fait son apparition dans La Symbolique du mal par l'entremise du Traité d'histoire des religions de Mircea Eliade, paru en français en $1949^{40}$. Le terme luimême constitue un outil commode pour unifier le champ des divers symboles religieux du mal, en les articulant de surcroît «à une compréhension intensive plutôt qu'extensive du mythe » : 
Le mal - souillure ou péché - est le point sensible et comme la «crise » de ce lien que le mythe explicite à sa façon; en nous limitant aux mythes concernant l'origine et la fin, nous avons chance d'accéder à une compréhension intensive plutôt qu'extensive du mythe. C'est en effet parce que le mal est l'expérience critique par excellence du sacré, que la menace de dissolution du lien de l'homme à son sacré fait ressentir avec la plus grande intensité la dépendance de l'homme aux forces de son sacrét ${ }^{41}$.

17 Au-delà de sa répétition maladroite, le terme "sacré " permet à Ricœur de formuler une hypothèse forte s'agissant des symboles du mal : d'une part le philosophe pose que les symboles du mal constituent un segment hautement significatif d'une théorie des mythes qui de surcroit limite efficacement l'analyse aux mythes d'origine et de fin du monde ; d'autre part il peut espérer comprendre le lien de l'homme au sacré par la crise même que constitue l'expérience du mal. Au passage, Ricœur rend compte du fait que cette crise est susceptible d'être surmontée, dans la mesure où la « réminiscence » des origines est d'ores et déjà solidaire, à ses yeux, d'une « expectation $»^{42}-$ autrement dit d'une espérance de délivrance du mal.

On a vu que le philosophe désigne d'emblée l'importance du mythe de chute adamique. Loin de constituer l'origine absolue de l'expérience biblique du mal, le mythe adamique narré dans la Genèse constitue déjà une reprise de cette expérience du mal, et plus précisément du péché, qui a pris forme sans lui. Il est très important pour Ricœur de faire comprendre que les auteurs bibliques n'ont aucunement thématisé l'expérience du péché en lien avec le récit de la chute adamique, car le mythe biblique de la chute exprime le fond d'une expérience pénitentielle qui lui préexiste. Contrairement au babylonien pour qui les dieux ne sont pas nécessairement justes, au grec pour qui les dieux sont parfois franchement mauvais, le Juif des temps bibliques se reconnaît pécheur face à un Dieu qui est seul Saint. Ce récit présuppose donc une expérience du péché mais ne lui a pas donné naissance : « Loin que le mythe adamique soit le point de départ de son expérience du péché et de la culpabilité [du peuple juif], il présuppose cette expérience et en exprime la maturité $»^{43}$. L'exégèse actuelle estime d'ailleurs que c'est l'expérience de la transgression de l'alliance qui constitue le péché proprement biblique.

Ricœur souligne l'importance de l'apôtre Paul : c'est lui qui a élevé Adam au rang de figure (tupos) du Christ ${ }^{44}$, de sorte que "l'événement" du Christ transformait rétroactivement en "événement" similaire la chute d'Adam; par un effet de choc en retour l'historicité du second Adam [Christ] conférait au premier Adam une historicité comparable et d'abord une individualité semblable à celle du Christ $»^{45}$. Adam n'est donc devenu une figure historique qu'à la faveur d'une lecture typologique, ce qui (notons-le au passage) est conforme au jugement de Ricœur selon lequel l'événementChrist (son message, sa mort, l'annonce de sa résurrection) n'a pu être rendu intelligible que par le moyen d'une réinterprétation des Écritures ${ }^{46}$. Cette analyse lumineuse n'empêche pas qu'aient existé dans la littérature intertestamentaire, donc avant Paul, des spéculations sur Adam portant notamment sur la durée de sa vie en Éden (sept années) ${ }^{47}$, que Ricœur ignorait vraisemblablement. Quoi qu'il en soit, Ricœur est fondé à dire que l'expérience de pénitence demeure bien antérieure à la spéculation sur Adam, puisqu'elle se disait déjà dans les psaumes de pénitence à vocation cultuelle. Il est à cet égard tout à fait symptomatique que le philosophe, parce qu'il vise la " "répétition" de l'expérience que le mythe explicite " spéculation religieuse - toujours peu ou prou teintée de gnosticisme - mais de 
l'expérience même du mal, tenue pour intelligible en raison de sa nature langagière. Avant tout mythe, a fortiori avant toute spéculation sur le mythe, l'expérience du mal trouve son mode d'expression dans le langage de l'aveu, «de part en part symbolique $»^{49}$. De la culpabilité individuelle - symbole du mal le plus récent - à celui de péché dont il est issu, jusqu'à l'archaïque souillure qui les a tous deux précédés, c'est encore "dans l'élément du langage $"^{50}$ que se meut la reprise philosophique.

La reprise philosophique de la "conscience de faute $»^{51}$ serait d'ailleurs impossible s'il n'existait la conviction que ce tout ce qui est langage est en soi compréhensible : même l'expérience du mal - tout ensemble expérience de cécité, d'équivocité, de scandale s'offre à la compréhension puisqu'elle s'articule dans le langage de l'aveu, ou plus précisément celui de la confession du mal. On pourrait dire d'une autre manière que l'on n'accède jamais à la faute, opaque en elle-même, que par l'aveu qui tire la confusion de l'émotion vers la «lumière de la parole $»^{52}$. Le lecteur trouve ici l'origine d'un geste éminemment ricœurien consistant à rechercher dans le langage même l'intelligibilité d'une réalité humaine. Dans La Symbolique du mal sourd la conviction que «par l'aveu l'homme reste parole jusque dans l'expérience de son absurdité, de sa souffrance, de son angoisse $»^{53}$. Ricœur atteste sa foi dans le langage et montre toute la portée et la puissance heuristique du tournant herméneutique de sa philosophie. Il n'est rien que l'humain ne trouve les ressources d'exprimer, serait-ce l'incompréhensibilité du mal, l'incapacité à l'empêcher et l'impuissance à y contribuer qui aboutissent au sentiment d'« aliénation à soi-même " ${ }^{54}$ au cœur de l'expérience du mal.

21 Revenons à l'ancrage de la théorie du symbole dans la phénoménologie du sacré, qui fait l'objet d'une rapide mise au point s'appuyant sur une esquisse du Traité d'histoire des religions d'Eliade, laquelle tient d'ailleurs presque entièrement dans la citation suivante :

[...] le symbolisme parlé renvoie ainsi aux manifestations du sacré, aux hiérophanies, où le sacré est montré dans un fragment du cosmos qui en retour perd ses limites concrètes, se charge de significations innombrables, intègre et unifie le plus grand nombre possible de secteurs de l'expérience anthropocosmique ; c'est donc d'abord le soleil, la lune, les eaux, c'est-à-dire des réalités cosmiques, qui sont symboles; dirons-nous alors que le symbole par son aspect cosmique est antérieur, voire étranger au langage ? Non point ; être symbole pour ces réalités c'est recueillir dans un nœud de présence une masse d'intentions significatives qui, avant de donner à penser, donnent à parler; la manifestation symbolique comme chose est une matrice de significations symboliques comme paroles ${ }^{55}$.

Ricœur concède ainsi à Eliade qu'il existe avant le symbolisme parlé un symbolisme sacré offert par les réalités physiques elles-mêmes. Le cas du ciel abordé au premier chapitre du Traité est à cet égard emblématique. Pour Eliade en effet, le ciel se révèle spontanément à qui le contemple comme infini, inaccessible, divin, sacré; il renvoie d'emblée l'humain à « tout autre chose " que son monde propre ${ }^{56}$. En lui-même, le ciel " "symbolise" la transcendance " ${ }^{57}$. Ricœur reprend donc d'Eliade l'idée d'un symbole cosmique. On aura toutefois remarqué que dans la citation précédente, le philosophe introduit un discret correctif au propos d'Eliade, par lequel il articule d'emblée le symbole hiérophanique à son expression symbolique. Il concède bien l'existence d'un " symbole-chose", mais pour indiquer que celui-ci ne se conçoit que comme " condensation d'un discours infini", comme "puissance d'innombrables symboles parlés $\aleph^{58}$. L'insertion de la théorie du symbole dans celle du sacré se fait donc sur la base étroite d'un symbolisme sacré qui a d'emblée besoin des symboles et des mythes 
qui signifient ce que les éléments naturels figurent seulement. La phénoménologie du sacré implique de fait une herméneutique des symboliques du mal, mais la réciproque n'est pas vraie, car force est de constater que dans le corps de l'ouvrage il est essentiellement question d'expérience pénitentielle en référence à des dieux et des théologies (babylonienne, tragique, hébraïque, orphique) sans aucune référence à la notion de sacré. Nous ne sommes pas en présence d'une phénoménologie des symboles du sacré, mais d'une ébauche de phénoménologie du sacré servant de prélude à une longue herméneutique des symboles religieux. L'usage du concept de sacré aura permis à Ricœur de prendre conscience de sa préférence marquée pour les «symboles parlés, et même uniquement les symboles du soi [...] déjà en voie de se couper des racines cosmiques du symbolisme $»^{59}$.

\section{L'epokhê dans l'Essai sur Freud}

Opérons un dernier saut, et reportons-nous par-delà de la Philosophie de la volonté à De l'interprétation (Essai sur Freud), ouvrage resté fameux en raison de la rigueur de la lecture ricœurienne de Freud. Ce n'est pas celle-ci qui nous intéresse au premier chef, mais seulement la discussion de la critique freudienne de la religion. Pour Ricœur, l'iconoclasme du freudisme en matière de religion est un fait; mais la possibilité de le dépasser en vue d'une foi éprouvée par la critique - une foi seconde - relève en revanche d'une intention explicitement affichée :

Mon hypothèse de travail, énoncée dès la Problématique ${ }^{60}$, est que la psychanalyse est nécessairement iconoclaste, indépendamment de la foi ou de la non-foi du psychanalyste, et que cette "destruction » de la religion peut être la contrepartie d'une foi purifiée de toute idolâtrie. La psychanalyse comme telle ne peut aller audelà de cette nécessité de l'iconoclasme. Cette nécessité ouvre sur une double possibilité : celle de la foi et celle de la non-foi, mais la décision entre ces deux possibilités ne lui appartient pas ${ }^{61}$.

Dans ce passage, on aura noté d'une part que la foi intervient au début, s'agissant la foi ou d'absence de foi du psychanalyste, et d'autre part qu'il convient a priori de la distinguer de la religion que détruit la perspective freudienne. Au-delà de cette critique, c'est encore la foi et la non-foi qui sont possibles; mais - c'est tout à fait remarquable - Ricœur dénie à la psychanalyse de la religion le droit de trancher en matière de foi : ni en sa faveur, ni en sa défaveur. Il faut donc comprendre que le concept de foi est formellement distinct de celui de religion. Mais de quelle foi s'agit-il ici? Ricœur n'élude pas la question, qui affirme en effet sans détour dès la problématique du livre I: «Le contraire du soupçon, dirai-je brutalement, c'est la foi $»^{62}$. Le propos indique que c'est plus précisément la foi du pari que Ricœur rattachait à la démarche anselmienne du croire pour comprendre ${ }^{63}$. En ce sens, c'est une foi plus large que la seule foi chrétienne, puisqu'elle caractérise selon Ricœur la démarche d'une phénoménologie du sacré, en tant que cette dernière suppose de participer - sur un mode neutralisé - à la croyance religieuse : «L'épochè exige [...] que je croie avec le croyant, mais sans poser absolument l'objet de sa croyance $»^{64}$ - objet que Ricœur, comme dans La Symbolique du mal, place sous le terme commode de sacré65.

Mais le fait même de se pencher sur un objet religieux n'est pas anodin : il relève pour Ricœur d'une forme d'attente que, « du sein de la compréhension, ce "quelque chose" [de sacré] s"'adresse" à moi " ${ }^{66}$. Il en va ici, fondamentalement, d'une foi dans le langage, et plus spécifiquement dans sa capacité à éclairer l'humain : 
Finalement, ce qui est implicite cette attente, c'est une confiance dans le langage; c'est la croyance que le langage qui porte les symboles est moins parlé par les hommes que parlé aux hommes, que les hommes sont nés au sein du langage, au milieu de la lumière du logos « qui éclaire tout homme en venant au monde ». C'est cette attente, c'est cette confiance, c'est cette croyance qui confèrent à l'étude des symboles sa gravité particulière. Je dois à la vérité de dire que c'est elle qui anime toute ma recherche. Or c'est elle qui est aujourd'hui contestée par tout le courant de l'herméneutique que nous placerons [...] sous le signe du "soupçon »; cette autre théorie de l'interprétation commence précisément par le doute qu'il y ait un tel objet $[. . .]^{67}$

Ricœur fait état ici de sa confiance et de son attente dans la capacité du langage à éclairer tout homme: que le propos s'apparente à une confession de foi dans la puissance du langage, c'est ce que vient indiquer le terme de "croyance», renforcée par la référence au prologue de l'évangile de Jean décrivant le Logos éclairant tout homme qui vient au monde $(J e a n ~ 1,9)^{68}$. Ce renvoi à un thème fondateur de la théologie biblique atteste que Ricœur puise dans sa culture biblique et théologique lorsqu'il thématise sa confiance dans le langage. Un lecteur peu féru de cette culture pourrait s'en offusquer : que signifie cette référence au logos johannique ? Doit-on comprendre qu'elle est centrale au propos? Inversement, une certaine connaissance de cette culture biblique permet d'interroger la citation de Ricœur. Celle-ci est en effet coupée et amenée de telle manière qu'il n'apparaît plus que le logos est l'incarnation de la parole même de Dieu, que l'évangéliste identifie avec la personne de Jésus. Il faudrait donc dire que d'un point de vue exégétique et théologique la citation de Ricœur équivaut à une déchristologisation, l'exaltation du langage préexistant étant célébrée à la place du logos johannique. Voilà qui est représentatif du style de pensée de Ricœur : ni strictement agnostique puisqu'il renvoie à des thèmes théologiques, ni théologique puisqu'il leur confère une dimension essentiellement profane. Dire la puissance du langage et non l'incarnation du Christ par le thème du logos, tel est le geste singulier de Ricœur. Ce n'est pas là un élément secondaire relevant de l'ornement rhétorique, car le philosophe renvoie encore à des éléments issus de la théologie chrétienne par l'idée d'une " attente d'une nouvelle Parole ", d'une " grâce de l'imagination » à l'œuvre dans le symbole; grâce dont Ricœur demande en outre si elle n'a pas « quelque chose à voir avec la Parole comme Révélation $»^{69}$.

C'est la raison pour laquelle l'approche ricœurienne de la religion fera à partir des années 70 une large place à l'herméneutique biblique. Celle-ci aura pour fonction de mettre en lumière la spécificité de la poétique biblique, laquelle est créditée d'une capacité à révéler (par exemple dans les paraboles) une dimension nouvelle de réalité et donc de l'existence. La phénoménologie de la religion se verra dès lors définitivement reléguée au second plan derrière l'herméneutique, étant « condamné[e] à passer par les fourches caudines d'une herméneutique, et plus précisément d'une herméneutique textuelle ou scripturaire $»^{70}$. Pour Ricœur, il s'avère impossible d'accéder phénoménologiquement à "l'immédiateté sauvage d'une expérience originaire $\aleph^{71}$, à celle de l'expérience religieuse moins qu'aucune autre. Derrière l'affirmation de ce principe méthodologique se dissimule peut-être une incapacité de Ricœur à sortir du langage religieux qui avait sa prédilection: convaincu qu'un philosophe ne peut jamais accéder au religieux que par la tradition religieuse à laquelle il appartient, Ricœur n'a malheureusement jamais confronté son herméneutique du langage biblique à d'autres herméneutiques religieuses, et n'a donc jamais visé quelque 
chose comme une expérience de la Transcendance par-delà la contingence des traditions religieuses

\section{NOTES}

1. Du texte à l'action. Essais d'herméneutique II, Paris, Seuil, 1986, p. 25 (Ricœur souligne; sauf mention expresse toutes les indications bibliographiques renvoient à Paul Ricœur).

2. E. Husserl, Idées directrices pour une phénoménologie, trad. par P. Ricœur, Paris, Gallimard, 1950, \$31, p. 100-101 pour les deux citations (Husserl souligne).

3. Sur la réduction eidétique comme saisie du "fait (Tatsache) dans son essence (eidos) », voir notamment « Kant et Husserl (1954) », À l'école de la phénoménologie, Paris, Vrin, 1986, p. 229.

4. E. Husserl, Idées directrices pour une phénoménologie, op.cit., \$58, p. 192.

5. Philosophie de la volonté. Tome I. Le Volontaire et l'Involontaire, Paris, Aubier, 1950, p. 7, pour les deux citations.

6. Ibid., p. 7 (Ricœur souligne).

7. Paris, Éd. du Temps Présent, 1948.

8. Philosophie de la volonté. Tome I, op.cit., p. 30.

9. Ibid., p. 33.

10. Ibid., p. 35 .

11. Ricœur a ainsi par exemple été président du mouvement du Christianisme social de 1958 à 1970.

12. Notre hypothèse de lecture dans notre monographie à paraître est en effet que Paul Ricœur a mis en œuvre une distinction qu'il a thématisée au sujet de l'enseignement: son œuvre philosophique relève d'une laïcité d'abstention, tandis que ses écrits chrétiens ressortissent d'une laïcité de confrontation. Cf. «Le protestantisme et la question scolaire », Foi Éducation, $\mathrm{n}^{\circ}$ 27, 1954, p.56 (une édition digitale de ce texte est accessible sur le site https:// bibnum.explore.psl.eu/s/psl/ dans le cadre d'un projet d'édition numérique que nous dirigeons, sous l'égide du Fonds Ricœur).

13. Philosophie de la volonté. II, Finitude et Culpabilité, t. 2 La Symbolique du mal, Paris, Aubier, 1960, p. 11. On notera la tonalité kierkegaardienne de ces deux termes.

14. Ibid., p. 323 (nous soulignons).

15. Ibid., p. 11.

16. "Culpabilité tragique et culpabilité biblique», Revue d'Histoire et de Philosophie religieuses 33, 1953, p. 297 (disponible sur https://bibnum.explore.psl.eu/s/psl/).

17. Ibid., p. 297 (pour les deux citations).

18. "L'homme de science et l'homme de foi (1952)", Le Semeur 51, 1952, p. 16.

19. On notera ici que déjà phénoménologie et herméneutique sont liées, la seconde étant une spécification de la première, ce qui préfigure l'importante thèse énoncée dans Du texte à l'action, selon laquelle d'une part "la phénoménologie reste l'indépassable présupposition de l'herméneutique. D'autre part, la phénoménologie ne peut se constituer elle-même sans une présupposition herméneutique (Du texte à l'action, op.cit., p. 40 ; Ricœur souligne).

20. La Symbolique du mal, op.cit., p. 27.

21. Ibid., p. 30.

22. Ibid., p. 26. 
23. Ibid., p. 326.

24. Ibid., p. 326.

25. Cf. M. Heidegger, Être et temps (1927) : « [...] voir dans ce cercle un cercle vicieux et chercher les moyens de l'éviter, ou même simplement l'“éprouver" comme une imperfection inévitable, cela signifie mécomprendre radicalement le comprendre. Ce dont il y va, ce n'est point d'ajuster le comprendre et l'explicitation à un idéal de connaissance qui n'est lui-même qu'une forme déchue du comprendre. Ce qui est décisif, ce n'est pas de sortir du cercle, c'est de s'y engager convenablement. » (trad. par E. Martineau, édition numérique hors commerce, § 32, 1985.)

26. La Symbolique du mal, op.cit., p. 285 (Ricœur souligne).

27. Ibid., p. 285.

28. Ibid., p. 14.

29. Ibid., p. 323.

30. Ibid., p. 287 (Ricœur souligne). C'est l'objet du dernier chapitre (« Le cycle des mythes », ibid., p. 285-321).

31. Ibid., p. 12.

32. "Le "Péché Originel": étude de signification", Le Conflit des interprétations. Essais d'herméneutique, Paris, Seuil, 1969, p. 265-282.

33. Ibid., p. 266.

34. Ibid., p. 266.

35. Ibid., p. 277.

36. "Herméneutique des symboles et réflexion philosophique", Le Conflit des interprétations, op.cit., p. 303.

37. La Symbolique du mal, op.cit., p. 324.

38. Ibid., p. 13.

39. Ibid., p. 13

40. Ricœur y renvoie tant dans l'introduction qu'en conclusion (ibid., p. 18, cf. p. 329). Il n'a pas été trompé par le titre (mal choisi, mais imposé par l'éditeur) puisqu'il discerne dans l'ouvrage non pas une histoire des religions, mais bien une "phénoménologie comparatiste » (ibid., p. 18) des hiérophanies.

41. Ibid., p. 13.

42. Ibid., p. 13 pour les deux citations.

43. Ibid., p. 222.

44. Voir Romains 5, 14 auquel Ricœur fait de toute évidence allusion ici.

45. La Symbolique du mal, op.cit., p. 14.

46. $C f$. « Préface à Bultmann » (Le Conflit des interprétations, op.cit., p. 373 sq).

47. Cf. le Livre des Jubilés retrouvé notamment à Qumrân en 1947 : A. Dupont-Sommer et M. Philonenko (dir.), La Bible. Écrits intertestamentaires, Paris, Gallimard, Bibl. de la Pléiade, 1987, p. 650.

48. La Symbolique du mal, op.cit., p. 14.

49. Ibid., p. 323.

50. Ibid., p. 16.

51. Ibid., p. 16.

52. Ibid., p. 15.

53. Ibid., p. 15.

54. Ibid., p. 15.

55. Ibid., p. 18 (Ricœur souligne).

56. M. Eliade, Traité d'histoire des religions (1949), Paris, Payot, 1990, p. 46.

57. Ibid., p. 46.

58. La Symbolique du mal, op.cit., 18 (pour les trois citations).

59. Ibid., p. 18. 
60. Voir De l'interprétation. Essai sur Freud, Paris, Seuil, 1965, p. 13-63.

61. Ibid., p. 226.

62. Ibid., p. 36.

63. Cf. ibid., p. 37.

64. Ibid., p. 38.

65. «Appelons "sacré" cet objet visé, sans préjuger de sa nature » (ibid., p. 37).

66. Ibid., p. 38 (Ricœur souligne).

67. Ibid., p. 38 (nous soulignons).

68. Le verset est sans doute cité de mémoire, la traduction n'étant pas tout à fait correcte, puisqu'elle mêle deux traductions qui sont également possibles : «C'était la véritable lumière qui éclaire tout homme venant au monde »; ou "C'était la véritable lumière qui, en venant dans le monde, éclaire tout homme » Jean, I, 9, trad. L. Segond mod.

69. De l'interprétation, op.cit., p. 44.

70. «Phénoménologie de la religion », in Lectures 3. Aux frontières de la philosophie, Paris, Seuil, 1994, p. 266.

71. Amour et Justice, Paris, Points Seuil, 2008, p. 48. 